\title{
The Effects of Serotonin Depletion and Raphe Grafts on Hippocampal Electrophysiology and Behavior
}

\author{
Gal Richter-Levin and Menahem Segal \\ Center for Neuroscience, The Weizmann Institute of Science, Rehovot 76100, Israel
}

The involvement of the serotonergic system in spatial learning and a possible correlation between serotonergic modulation of hippocampal electrical activity and spatial learning were studied in rats. Control, partial septal-lesioned (SL), 5,7-dihydroxytryptamine (5,7-DHT)-injected (DHT), doublelesioned (5,7-DHT and SL; DL), and DL rats that were transplanted with embryonic raphe grafts into the hippocampus (RG) were tested in a spatial task in a water maze and in an active avoidance shuttle-box task. The responses of the dentate gyrus (DG) to perforant-path (PP) stimulation were examined in the same rats, under the following conditions: (1) priming stimulation of the PP (testing feedback inhibition), (2) priming stimulation of the commissural pathway (testing feedforward inhibition), (3) during repeated stimulation of the PP at $7 \mathrm{~Hz}$ (frequency potentiation), and (4) following tetanic stimulation [long-term potentiation (LTP)].

DL, but not DHT or SL, treatment severely impaired the performance of rats in both reference- and working-memory tasks in the water maze.This effect was not seen in the shuttle box. The ability of the DG to exhibit LTP, which was reduced in the DHT and SL rats, was apparently similar to controls in the DL group. DL, but not DHT or SL alone, resulted in a reduction of inhibition in the DG. Both the behavioral deficits and the reduction in hippocampal inhibition were ameliorated by intrahippocampal raphe grafts.

These results indicate that the serotonergic innervation of the hippocampus plays a role in spatial learning when the septohippocampal neurotransmission systems are disrupted. Furthermore, these results suggest that restoration of modulation of hippocampal inhibition, by raphe grafts, underlies the behavioral recovery observed in these rats.

The hippocampus is involved in learning and memory processes and, in particular, in spatial learning (O'Keefe and Nadel, 1978; Morris et al., 1982; Barnes, 1988; Squire and Zola-Morgan, 1988). Serotonergic fibers, arising from the midbrain raphe (MR) nuclei, densely innervate the hippocampal formation and the dentate gyrus (DG; Azmitia and Segal, 1978; Moore, 1981), suggesting a role for 5-HT in hippocampal functions. Depletion of forebrain 5-HT, however, has little effect on spatial performance in rats (Asin et al., 1985; Altman et al., 1989; Richter-

\footnotetext{
Received Sept. 26, 1990; revised Dec. 26, 1990; accepted Dec. 28, 1990.

We wish to thank H. Markram and $M$. Wain for valuable comments on the manuscript and Ms. V. Greenberger for help in AChE staining and ${ }^{3} \mathrm{H}$-imipramine binding. This research was supported by a grant from MINERVA Foundation, Munich, Germany.

Correspondence should be addressed to Menahem Segal at the above address.

Copyright (c) 1991 Society for Neuroscience $0270-6474 / 91 / 111585-12 \$ 03.00 / 0$
}

Levin and Segal, 1989). Recent studies have indicated that, under conditions in which other neurotransmission systems are disrupted, the serotonergic system becomes crucial for correct spatial performance (Vanderwolf, 1987; Nilsson et al., 1988; Richter-Levin and Segal, 1989).

The perforant path (PP) is the main excitatory pathway innervating the hippocampus. The response of DG granule cells (GCs) to PP stimulation and the expression of long-term potentiation (LTP) of responses to this stimulation vary with the behavioral state of the animal (Vanderwolf, 1988; Austin et al., 1989; Bramham and Srebro, 1989; Sharp et al., 1989). Voluntary behaviors are associated with an atropine-resistant $\theta$-activity (rhythmical slow activity) that is dependent on the integrity of the serotonergic innervation of the hippocampus (Vanderwolf, 1988). During this type of rhythmical slow activity, spontaneous hippocampal EEG spikes are suppressed (Vanderwolf, 1988). We have recently found that 5 -HT-releasing drugs can modulate the responsiveness of DG GCs to PP stimulation as well as suppress hippocampal EEG spikes (Richter-Levin and Segal, 1990a). Furthermore, MR cells grafted into the hippocampus can restore hippocampal response to application of these drugs in 5-HT-depleted rats (Richter-Levin and Segal, 1990b).

The present study was designed to analyze the nature of the serotonergic involvement in learning and memory processes related to hippocampal activity. The effects of 5-HT depletion in septal-lesioned (SL) rats were examined at behavioral and physiological levels. At the behavioral level, experiments were conducted with a spatial memory task and with an associative learning task that is not assumed to depend on hippocampal integrity. At the physiological level, DG responses to PP stimulation were studied by analyzing feedforward and feedback inhibition, frequency potentiation (Andersen and Lomo, 1967), and LTP. Finally, we tested the possible restoration of these impaired behavioral and physiological functions, by an intrahippocampal MR graft.

The results indicate that the serotonergic innervation of the hippocampus modulates hippocampal responses to afferent stimulation and suggest that this modulation becomes crucial for spatial memory when the cholinergic input is reduced.

\section{Materials and Methods}

Subjects

Subjects were male Wistar rats from a local breeding colony. Surgery and transplantations were carried out at the age of $6-8$ weeks. The rats were assigned randomly for control $(n=8)$ or to receive either septal lesions (SL; $n=8)$, 5,7-dihydroxytryptamine (5,7-DHT) injection (DHT; $n=9$ ), combined SL and 5,7-DHT [double lesion (DL); $n=9$ ), or DL plus a raphe graft into the hippocampus (RG; $n=9$ ). The rats were then housed in a temperature-controlled room, four or five per cage, 
with a 12:12-hr light/dark cycle and free access to food and water, for 4 months until the behavioral experiments commenced. Experiments lasted 4 months.

\section{Lesions}

\section{Serotonin depletion}

Bilateral intraventricular injections of 5,7-DHT [200 $\mu \mathrm{g}$ (free-base) in $20 \mu \mathrm{l}$ saline containing $0.2 \mathrm{mg} / \mathrm{ml}$ ascorbic acid] were administered to anesthetized rats (chloral hydrate, $3.5 \%$ solution, $10 \mathrm{ml} / \mathrm{kg}$, i.p.) 2 weeks before grafting.

\section{Septal lesions}

A bipolar $250-\mu \mathrm{m}$ stimulating electrode was placed stereotaxically in the medial septum. DC current $(3 \mathrm{~mA} / 30 \mathrm{sec})$ was used to electrolytically lesion the septum.

\section{Grafting}

The midline tegmentum (measuring about $1 \mathrm{~mm}^{3}$ ) of embryonic day 14 (E14) einbryos was dissected out for raphe grafts, as previously described (Segal and Azmitia, 1986). Tissue was mechanically dissociated, and a total volume of $1 \mu \mathrm{l}$ was injected bilaterally into each host hippocampus.

\section{Behavioral tests}

Behavioral tests included an active avoidance task and a spatial-memory water-maze task.

\section{The avoidance task}

The active avoidance task was measured in a shuttle box. The shuttle box consists of two compartments. In order to avoid or escape an electric foot shock, the animal had to move from one compartment to the other when a 40-sec tone [conditioned stimulus (CS)] was sounded. Ten seconds after the onset of the tone, an electric shock was applied through the grid floor for $30 \mathrm{sec}$. Shock intensity was set to a level at which al rats startled. Forty-five trials were given in a session, with intertrial intervals of $40 \mathrm{sec}$.

\section{The water-maze task}

A reference-memory test. Rats were placed in a circular pool of water (130-cm diameter, $50-\mathrm{cm}$-high rim, and water level of $15 \mathrm{~cm})$, to which milk powder had been added to make the water opaque, and allowed to swim freely for a maximum of $1 \mathrm{~min}$ or until they reached a hidden platform (top surface: $10 \mathrm{~cm}$ in diameter, $3 \mathrm{~cm}$ below water level). At the end of each trial, rats were either led to or left on the platform for $15 \mathrm{sec}$. Rats were given three blocks of two trials each day for three consecutive days. On the fourth day, the platform was moved to a new position, and the rats were given four more trials to locate the platform. Their actual path was hand drawn and was later analyzed as follows: the water maze was divided into four quadrants. The number of times a rat crossed into or out of a circle centered in each quadrant was used as an estimate of the time this rat had spent in that quadrant.

A working-memory test. Two weeks later, the same rats were tested again and given only two trials per day for $6 \mathrm{~d}$. Each day the platform was placed in a different location. The difference in escape time, between the first and the second trials on each day, was used as a measure of working memory.

\section{Electrophysiology}

A bipolar $125-\mu \mathrm{m}$ stimulating electrode was implanted in the PP (coordinates: $8 \mathrm{~mm}$ posterior to bregma, $4.2 \mathrm{~mm}$ lateral; depth adjusted to yield maximal response of the DG) and into the contralateral DG (coordinates: $4 \mathrm{~mm}$ posterior to bregma, $2.5 \mathrm{~mm}$ lateral to the midline; depth, $3.5 \mathrm{~mm}$ ). A recording electrode (glass microelectrode; tip diameter, $2-5 \mu \mathrm{m}$; filled with $3 \mathrm{M} \mathrm{NaCl}$ ) was placed in the DG [coordinates: $4 \mathrm{~mm}$ posterior to bregma, $2.5 \mathrm{~mm}$ lateral; depth adjusted to yield largest excitatory postsynaptic potential (EPSP) response to stimulation of the PP]. Thirty minutes later, the stimulus-pulse duration was adjusted to yield a population spike (PS) threshold at $0.25 \mathrm{~mA}$. Stimulus intensity of $0.35 \mathrm{~mA}$ was then used to obtain measurements of feedback and feedforward inhibition. Field potentials were amplified, filtered $(0.1-10 \mathrm{KHz})$, and stored on floppy disks for off-line analysis. PS and
EPSP slope were measured as described previously (Richter-Levin and Segal, 1990a). The following sequence of stimulation patterns was used:

\section{Feedback inhibition}

A twin-pulse PP stimulus was delivered at increasing interpulse intervals $(30-150 \mathrm{msec})$. Feedback inhibition was evaluated as the ratio of the size of the second to the first PS.

\section{Feedforward inhibition}

The DG commissural pathway was activated by stimulating the contralateral DG at different intervals prior to PP stimulation (10-150 $\mathrm{msec}$ ). Feedforward inhibition was evaluated as the ratio of the size of the response to PP stimulation after commissural stimulation and that of PP stimulation of the same intensity with no priming stimulation.

\section{Frequency potentiation}

The PP was stimulated for $30 \mathrm{sec}$ at $7 \mathrm{~Hz}$. The response of the DG was recorded on a digital oscilloscope at the end of the $30 \mathrm{sec}$, and PS size was measured manually from the oscilloscope.

\section{Long-term potentiation}

One hour after the frequency potentiation test, a possible LTP was examined. Tetanic stimulation consisted of 10 trains of 10 pulses at $400 \mathrm{~Hz}$, with $1-\mathrm{sec}$ intervals between trains. Pulse intensity was 0.35 $\mathrm{mA}$. Input-output curves were recorded before and $30 \mathrm{~min}$ after tetanic stimulation. LTP was measured as the difference in EPSP slope and PS size before and $30 \mathrm{~min}$ after the tetanic stimulation.

\section{Histology}

At the end of the recording session, the rats were killed, and their brains were quickly removed and kept at $-70^{\circ} \mathrm{C}$. Brains were sliced into $30-$ $\mu \mathrm{m}$ sections at $-20^{\circ} \mathrm{C}$ and then stained for cells (cresyl violet), cholinergic fibers (AChE; Biegon and Wolff, 1986), and serotonin terminals (using ${ }^{3} \mathrm{H}$-imipramine binding; Gross-Isseroff and Biegon, 1988). Quantitative analyses of $\mathrm{AChE}$ content and of ${ }^{3} \mathrm{H}$-imipramine binding were carried out using a videocamera-based computerized image analysis system (Isseroff and Lancet, 1985).

\section{Analysis}

Results are presented as mean \pm SEM. For statistical comparison, ANOVA for repeated measurements, $t$ tests, or Pearson correlation coefficients were used as indicated.

\section{Results}

\section{Histological analysis}

Histology

The extent of the septal lesion was assessed by an independent observer and was rated as 1 (intact septum), 2 (a partial lesion), or 3 (complete lesion). In all rats with septal lesions, the severity of the lesions was similar (SL, $2.2 \pm 0.1 ; \mathrm{DL}, 2.2 \pm 0.1 ; \mathrm{RG}$, $2.3 \pm 0.1)$. The regions completely destroyed by the lesion included the caudal medial septum, the vertical limb of the diagonal band, the anterior commissure, and the precommissural fornix (Fig. 1A). Partial damage was afflicted to the anterior medial and lateral septal nuclei.

Grafted tissue could easily be identified in cresyl violet-stained brain sections (Fig. $1 B$ ) from all eight grafted rats $(n=8)$. In six of these rats, the grafts were located in the ventromedial sector of the hippocampus. In the other two rats, the grafts were localized in the ventricles bordering the hippocampus, and attached to the DG.

\section{AChE staining}

Levels of AChE staining were compared in four areas of the hippocampus: DG, CA1, CA3, and CA4. In all four areas, in 

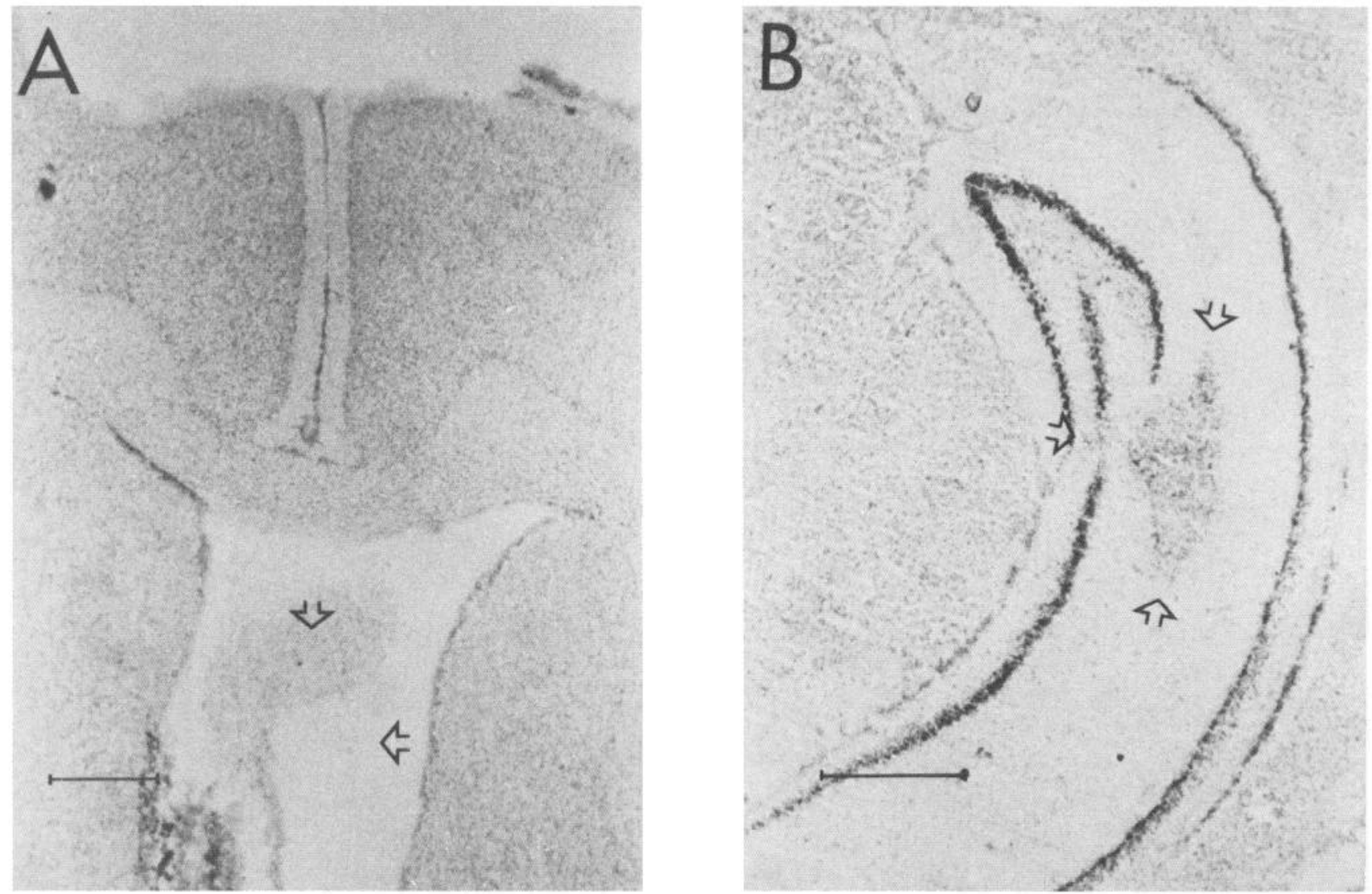

Figure 1. Cresyl violet sections of a septal lesion $(A)$ and a raphe graft $(B)$. Arrows indicate the lesioned area and the graft. Scale bars, $1 \mathrm{~mm}$.

all septal-lesioned groups (SL, DL, and RG), there was a significant reduction in AChE staining, as compared to controls (Fig. 2). There was also a reduction in AChE in the DHT group, but to a lesser extent (Fig. 2). The reason for this is not clear and is currently being studied. It is, however, important to note that MR grafts into the hippocampus did not cause any elevation of the AChE staining in the hippocampus above the level of the DL group, and thus, any effects that these grafts did have were not due to compensation for this reduction in AChE staining.

\section{${ }^{3} \mathrm{H}$-imipramine binding}

${ }^{3} \mathrm{H}$-imipramine binding was measured in the hippocampus in order to assess the 5,7-DHT-induced damage to serotonergic fibers and its restoration by the graft (Fig. 3). ${ }^{3} \mathrm{H}$-imipramine binding was compared at $1 \mathrm{~mm}$ and at $2 \mathrm{~mm}$ away (lateral and medial to the graft) from the graft (Fig. 4a). The amount of binding $2 \mathrm{~mm}$ from the graft was used for comparison with the other groups (Fig. $4 b$ ). ${ }^{3} \mathrm{H}$-imipramine binding in lesioned rats (DHT, DL) was significantly lower than in controls (ANOVA, $p<0.05$; Figs. $3,4 b$ ). A reduction was also noticed in the SL group, but ${ }^{3} \mathrm{H}$-imipramine binding in this group was still significantly higher than that in the DHT and DL rats. In the grafted rats $(\mathrm{RG}),{ }^{3} \mathrm{H}$-imipramine binding was significantly higher than in lesioned rats without grafts (ANOVA, $p<0.05$; Figs. 3, $4 b$ ).

\section{Behavioral studies}

\section{Active avoidance task}

All 5-HT-depleted groups (DHT, DL, RG) performed better than the control or SL groups (Fig. 5). The average number of avoidances (of a maximum of 45) in these groups was $22 \pm 0.8$, compared to only $11 \pm 0.5$ in control and SL groups (ANOVA, $P<0.008)$. Though septal lesions are reported to enhance the performance of rats in such tasks (King, 1958), no enhancement was observed in the SL rats. This was probably because these rats were only partially lesioned. The combination of 5-HT

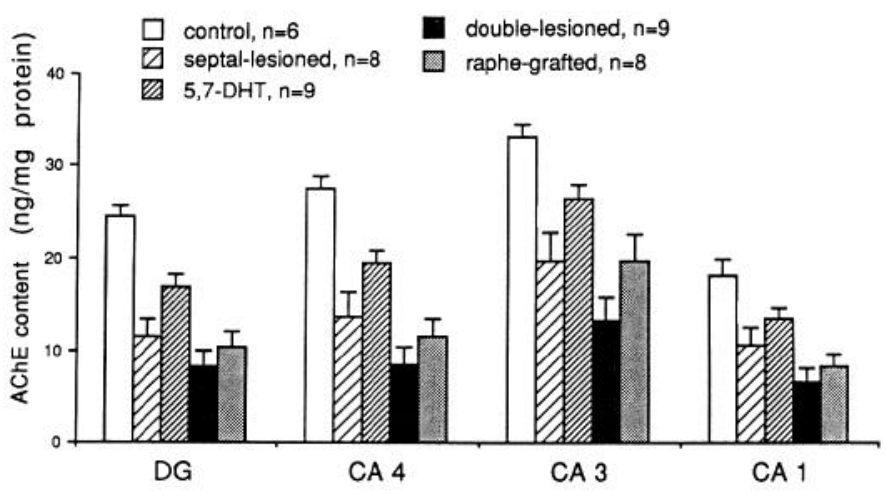

Figure 2. AChE content was measured in four areas in the hippocampus: DG, CA4, CA3, and CA1, using an image analysis system. Gray level was transformed into $\mathrm{ng} / \mathrm{mg}$ protein using a standard curve. There is a significant reduction in AChE content in the SL, DL, and RG groups (all are septal lesioned) as compared to the control (ANOVA, $P<0.05$ ). The largest differences are in the DG and CA4. Also noticed in the DHT group was a small reduction to a level that was still significantly higher than in the other lesioned groups (ANOVA, $P<0.05$ ) except CA1. Error bars represent SEM. 

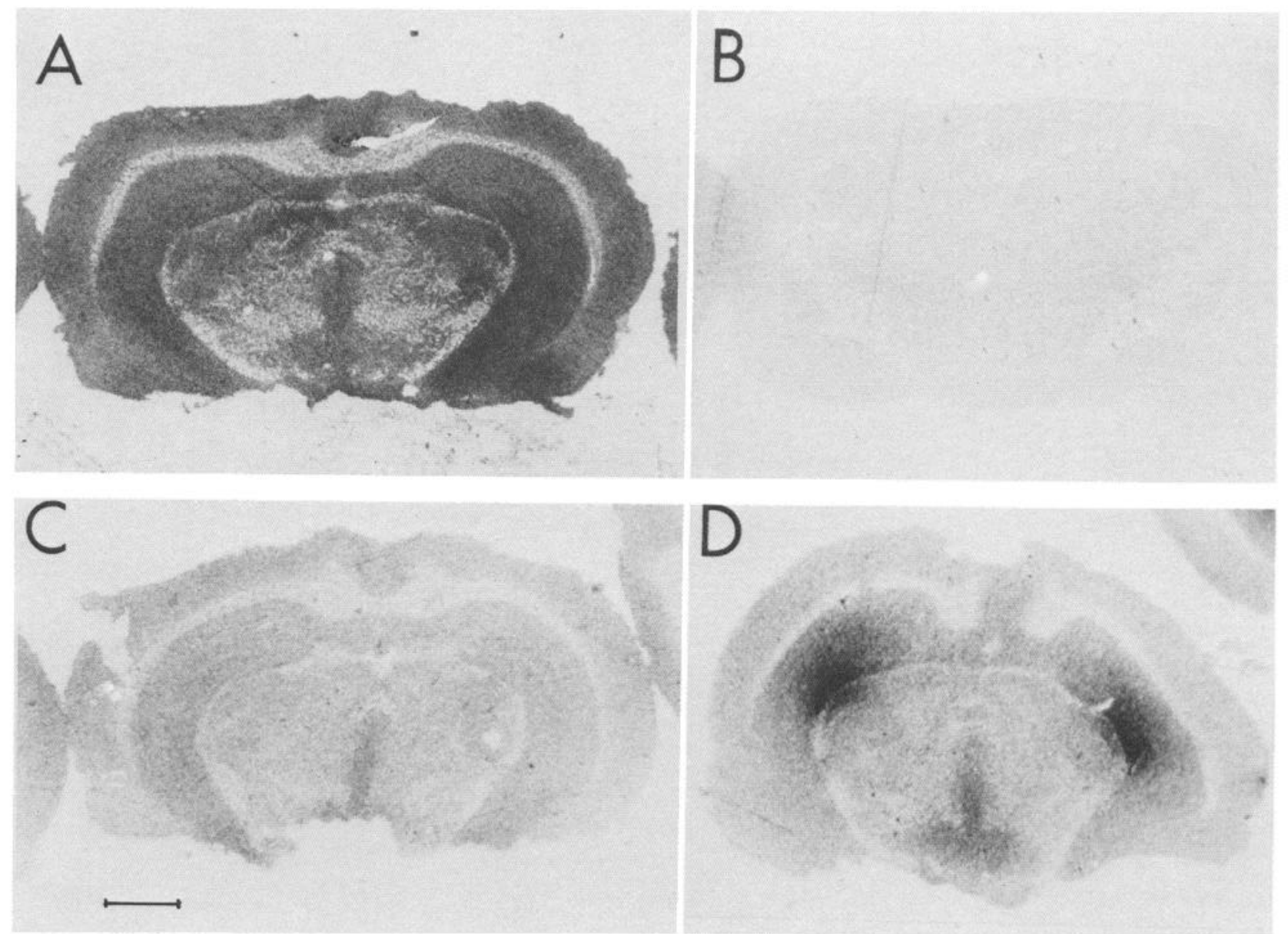

Figure 3. ${ }^{3} \mathrm{H}$-imipramine binding in control brain $(A)$, in the presence of $1 \mathrm{~mm}$ amitriptyline (for nonspecific binding; $B$ ), in DHT-treated brain $(C)$, and in RG $(D)$. Scale bar, $2 \mathrm{~mm}$.

depletion and septal lesion did not contribute to or modify the performance of this group compared to the DHT group. Furthermore, raphe grafts, located in the hippocampus, had no effect on the performance of 5-HT-depleted rats in the shuttle box.

\section{The water maze}

Reference memory. Depletion of forebrain 5-HT had a small but significant adverse effect on the performance of rats in the reference-memory water-maze task (DHT vs. control: $F=4.65$, $p<0.03$, ANOVA for repeated measurements; Fig. 6). However, when combined with a septal lesion, which by itself had no effect, 5-HT depletion severely affected the ability of these rats to perform in the maze (DHT vs. DL: $F=4.37, p<0.04$ ). DL rats that had received raphe grafts exhibited significantly better water-maze performance (DL vs. RG: $F=7.21, p<0.01$; Fig. 6).

Quadrant analysis. A difference in search strategy was observed between the DL group and all other groups. The DL group tended to exhibit a "random search" characterized by swimming in all four quadrants (Fig. 7a1), whereas the other groups showed an "oriented search" (Fig. 7a2), characterized by swimming toward the platform. To quantify this observation, the position of the invisible platform was shifted after the tenth block (Fig. 7a1,2), and the behavior of the rat in the four quadrants of the water maze was analyzed. Rats with an oriented search strategy spent significantly more time in the third quadrant (the previous location of the platform) than in the other three quadrants (control, SL, and DHT: $63.5 \pm 0.8 \%$ of total crossing). Those with a random search strategy (the DL group) spent about equal time in each quadrant (only $32 \pm 6.7 \%$ of total crossing in the third quadrant; ANOVA, $p<0.002$; Fig. $7 b$ ). The RG group was similar to control. Relatively less time was spent by all groups in the fourth quadrant because this was the new platform location, and once the rats entered this quadrant, they were usually able to locate the platform.

Working memory. After the rats became familiar with the water maze and the platform (reference memory), the position of the platform was changed daily (Fig. $8 a 1$ ), and rats had to memorize the new platform location (working memory). Control rats tended to search for the platform in the first trial and to swim directly to the new location of the platform on the second trial (Fig. 8a2). The SL and the DHT groups showed the same tendency but with somewhat higher variability (Fig. $8 b$; control vs. SL: $F=4.58, p<0.038$; control vs. DHT: $F=$ $4.78, p<0.035$; ANOVA for repeated measurements). In contrast, the DL rats, searching randomly, showed no improvement on the second trial (Fig. $8 a 3, b$; control vs. DL: $F=11.65, p<$ $0.0016)$. The RG group showed a significantly better performance when compared to DL rats (DL vs. RG: $F=5.84, p<$ 0.02; Fig. 8b).

We have examined a possible correlation between the per- 
a

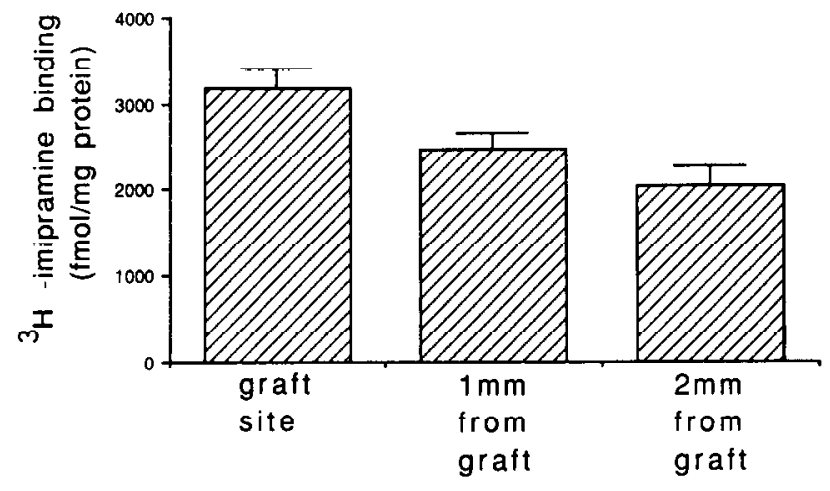

b

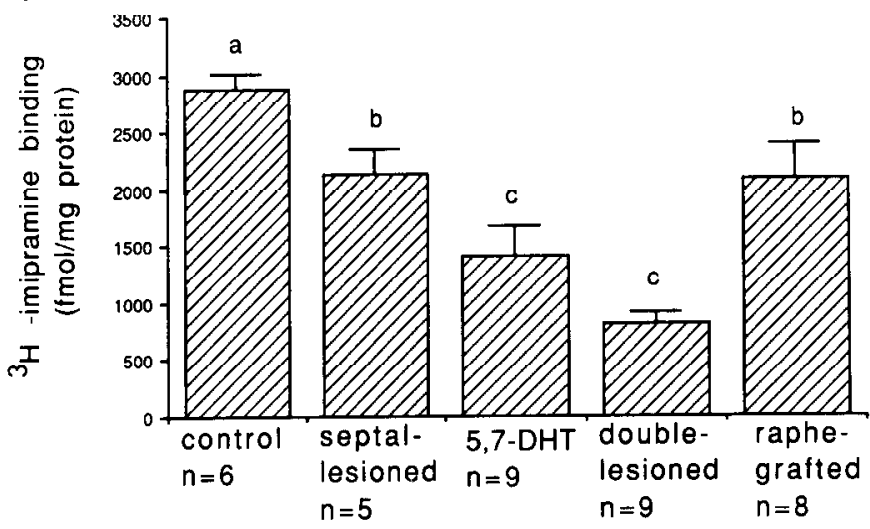

Figure 4. $a,{ }^{3} \mathrm{H}$-imipramine binding in grafted animals, at different distances from the graft site. Binding was assessed using an image analysis system. $b,{ }^{3} \mathrm{H}$-imipramine binding: comparison between groups. For the grafted group, binding $2 \mathrm{~mm}$ from the graft site was taken for comparison. Significant differences between groups are indicated by differences in letters $(a-c$; ANOVA, $P<0.05)$. Error bars represent SEM.

formance in the working-memory water-maze task and $\mathrm{AChE}$ levels. There was a low but significant correlation coefficient when all rats were grouped together $(r=-0.52 ; p<0.0001 ; n$ $=48$ ), indicating that the reduction in $\mathrm{AChE}$ can explain part of the variability in performance observed among the different gruups. The correlation was higher when the DL group was tested alone $(r=-0.82 ; p<0.003 ; n=10)$, indicating that 5-HT may have an important role in spatial working memory, in that when it is absent, the integrity of the cholinergic innervation becomes crucial and determines the ability of the rat to perform in this task. These results also suggest a possible interaction between the cholinergic and serotonergic lesions that could underlie the behavioral deficits in these rats.

\section{Electrophysiology}

Feedback inhibition

A complete inhibition of the response to a second stimulation of the PP was observed up to interpulse interval of $30 \mathrm{msec}$ (Fig. 9a), which weakened gradually until no longer significant at interpulse interval of $90 \mathrm{msec}$. In general, variation among animals within groups was very high, and it may have masked

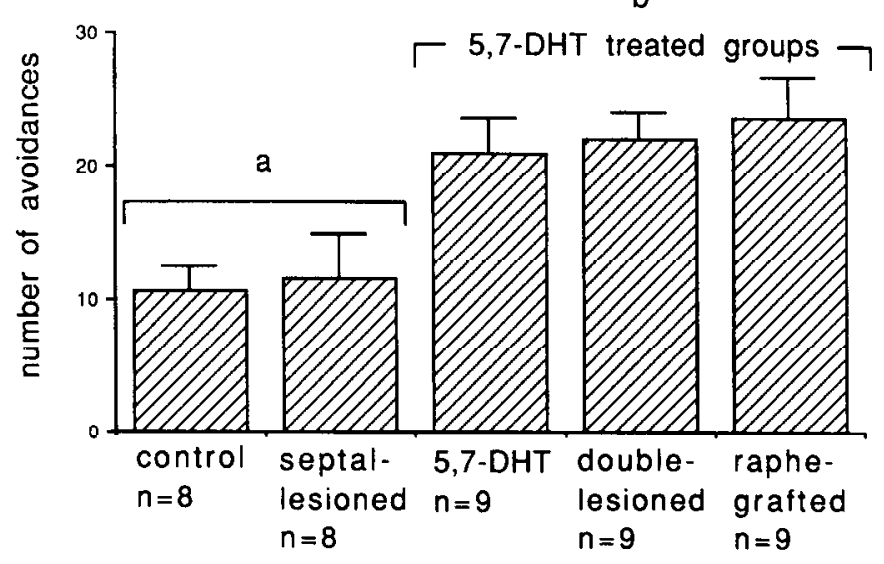

Figure 5. Performance in the active avoidance task. Rats were given 45 trials, and the number of times that they avoided the shock, by moving to the other compartment of the shuttle box, was automatically monitored. The averaged number of avoidances for each group \pm SEM is shown (CS duration, $40 \mathrm{sec}$; US duration, $30 \mathrm{sec}$; CS-US interval, 10 $\mathrm{sec}$; intertrial interval, $40 \mathrm{sec}$ ). All rats pretreated with 5,7-DHT (b) have performed significantly better than the control or septal-lesioned rats $(a$; ANOVA, $P<0.002)$. Raphe grafts had no effect on performance of this task. Error bars represent SEM.

differences between groups. Thus, though a tendency to increased inhibition was noticed in the SL and DHT groups, this was not significant (Fig. $9 b$ ). This tendency to increased inhibition, however, did not exist in the DL group.

\section{Feedforward inhibition}

The response to PP stimulation was inhibited by priming stimulation of the commissural path up to interpulse interval of 60 msec (Fig. 10a). 5-HT depletion or septal lesion did not alter feedforward inhibition. In contrast, in the DL rats, feedforward inhibition, at inter pulse inter vals of 10 and $30 \mathrm{msec}$, was blocked (ANOVA, DL vs. all other groups: $F=4.46, P<0.007 ; F=$ $4.99, P<0.003$, respectively; Fig. $10 b$ ). The presence of raphe grafts in the hippocampus restored the inhibitory effects of commissural stimulation on the response of the DG to activation of the PP (Fig. 10b). There was a low but significant correlation

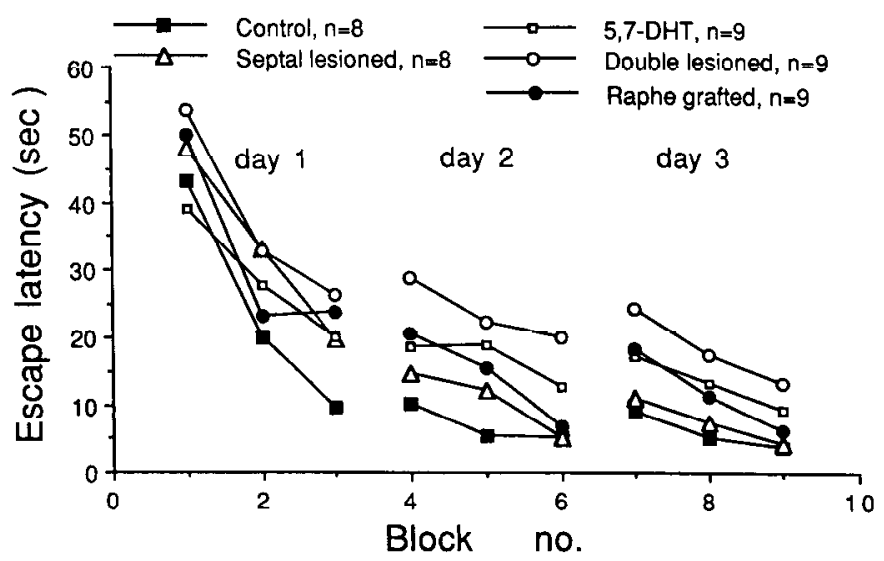

Figure 6 . The water-maze task-a reference-memory procedure: latencies to escape to a fixed-position hidden platform. Comparison of the escape latencies of groups over $3 \mathrm{~d}$, ANOVA for repeated measurements: control versus 5,7-DHT, $F=4.65, p<0.03$; DL versus 5,7DHT, $F=4.37, p<0.04$; DL versus RG, $F=7.21, p<0.01$. 
$\underline{\mathrm{a}}$
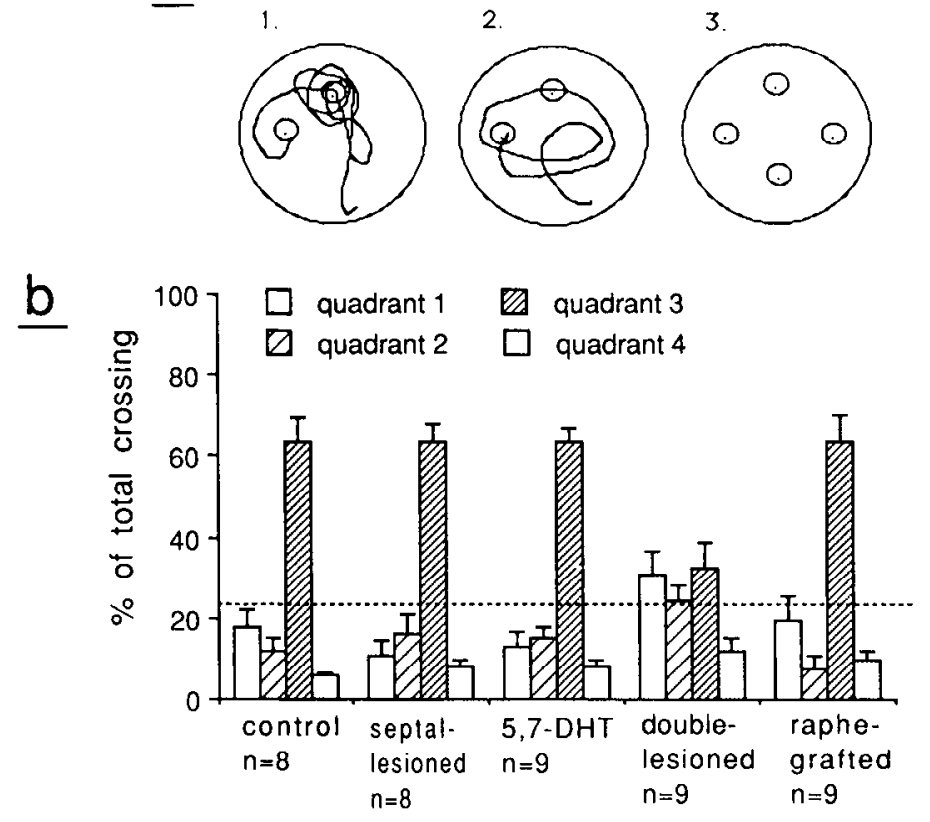

Figure 7. Quadrant analysis. After the ninth block of trials (see Fig. 6), the platform was shifted to a new location (depicted in schematic diagram of water maze, $a, 1$ and 2). Control rats tended to swim to the previous location of the platform, as illustrated in the representative example above $(a l)$. This type of search was referred to as an "oriented search." In contrast, the DL rats tended to search randomly for the platform (a2). To quantify this observation, the water maze was divided into four quadrants $(a 3)$, the actual path of each rat was analyzed manually, and the number of times a rat crossed into or out of a circle centered in each quadrant was used as an estimatc of the time this rat had spent in each quadrant $(b)$. Rats with an oriented search strategy tended to spend more than $60 \%$ of the time in the third quadrant (the previous location of the platform). The DL rats spent significantly less time in this quadrant (ANOVA, $P<0.002$ ) and tended to spend equal time in each quadrant ( $b$, dashed line). Error bars represent SEM. $\underline{a}$

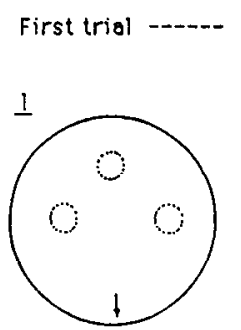

Second trial
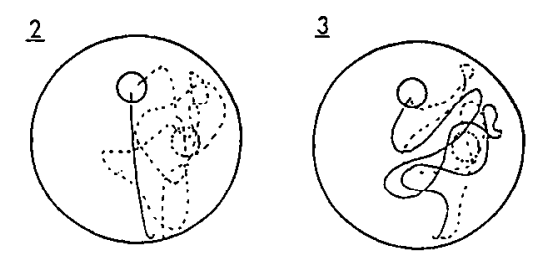

$\underline{b}$

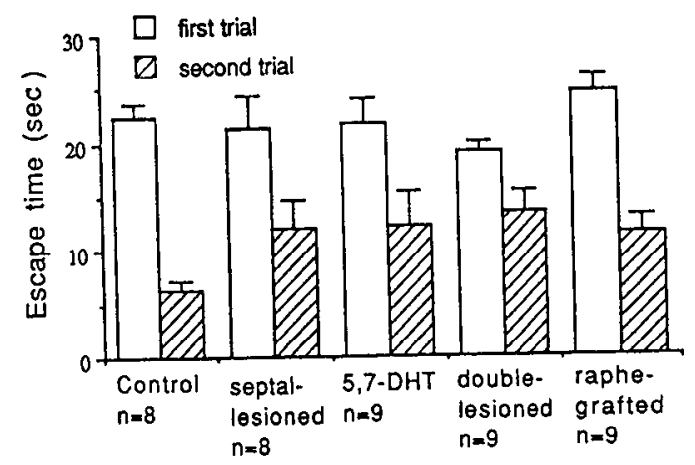

Figure 8 . The water-maze task-a working-memory procedure: rats were given two trials per day, for $6 \mathrm{~d}$, and the difference in escape latency between the first and second trials was used to assess working memory. $a: 1$, Threc possible locations of the platform. 2 and 3, Representative examples of performance of control (2) and DL (3) rats. $b$, The averaged escape latencies on the first and second trials of all groups over the $6 \mathrm{~d}$ of the test. Comparison of the differences in escape latencies between the first and the second trials of groups over $6 \mathrm{~d}$, ANOVA for repeated measurements: control versus $5,7-\mathrm{DHT}, F=4.78, p<0.03$; control versus SL, $F=4.58, p<0.03$; control versus $\mathrm{DL}, F=11.65, p<0.001$; DL versus RG, $F=5.84, p<0.02$. Error bars represent SEM. between the level of feedforward inhibition and the performance of the rats in the reference-type water-maze task $(r=-0.52 ; p$ $<0.001 ; n=36$ ), as well as between the performance in the working-memory task and the level of feedforward inhibition $(r=-0.40 ; p<0.01 ; n=36)$.

\section{Frequency potentiation}

A large increase (200-400\%) in PS size was observed $30 \mathrm{sec}$ after the beginning of stimulation. An increased number of failures to elicit frequency potentiation was observed in the 5-HTdepleted group (Fig. $11 a, b ; \chi^{2}$ test, $p<0.02$ ). This reduction in probability of frequency potentiation was not seen in the DL group. When failing to be potentiated, the field potential disappeared during the train of the stimulation, but was fully restored immediately after cessation of stimulation.

\section{Long-term potentiation}

EPSP slope. In control rats, tetanic stimulation of the PP causcd a consistent increase in EPSP slope. In both DHT and SL groups, the same stimulation was less effective in producing LTP (ANOVA for repeated measurements; control vs. DHT: $F=3.2, p<$ 0.08 ; control vs. SL: $F=7.7, p<0.009$; Fig. $12 b, c, f)$. In contrast, LTP was similar in the DL and the control groups and significantly different from that of the DHT $(F=8.9 ; p<0.005)$ and the SL $(F=16.1 ; p<0.0003)$ groups. LTP in RG rats was similar to single-lesioned groups (DHT and SL) and significantly different from both control $(F=4.3 ; p<0.04)$ and $\mathrm{DL}(F=$ $10.6 ; p<0.003$ ) rats.

Population spike size. Although more variable, the same tendency as with EPSP slope was observed when PS size was measured. One control rat did not exhibit any PS potentiation, and thus differences between the single-lesioned (DHT and SL), RG, and control groups were not statistically significant. The DL 


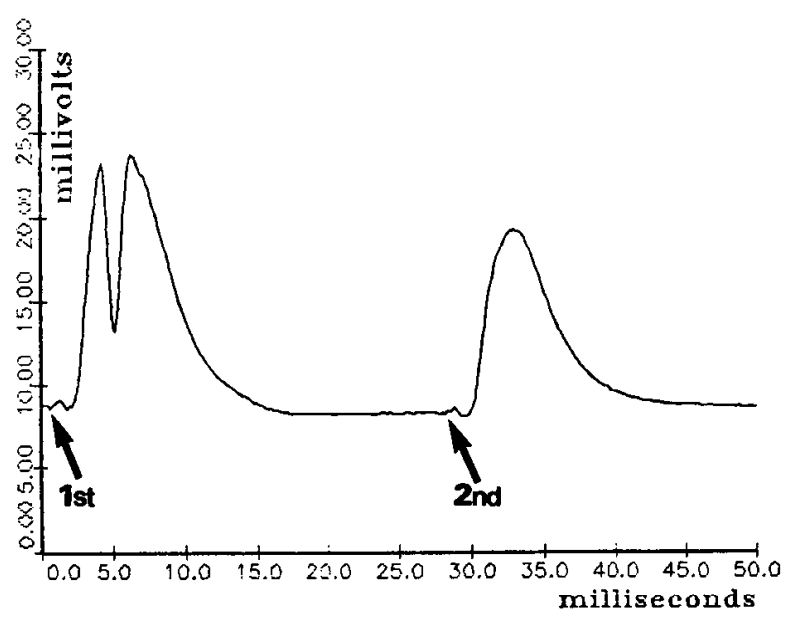

b

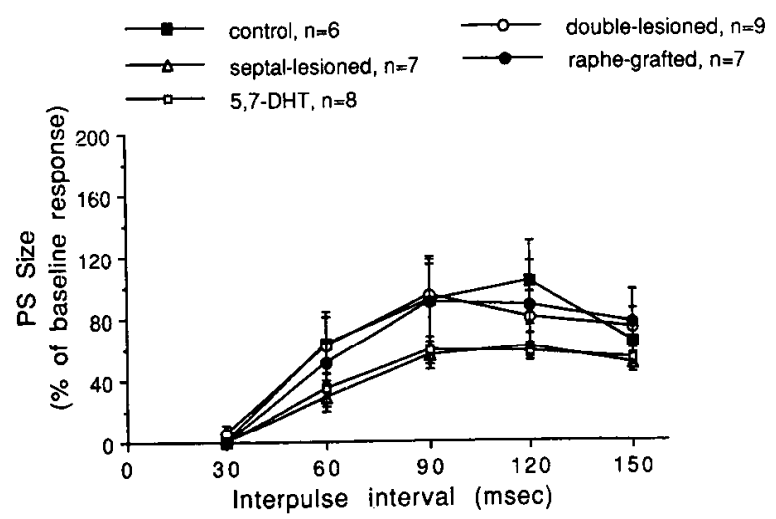

Figure 9. Feedback inhibition. $a$, The response to a second activation of the PP (2nd) is inhibited by priming activation (1st) of the same pathway. $b$, Comparison of feedback inhibition between groups. Inhibition was measured as the relative decrease in response to a second activation compared to priming activation of the PP. There was no clear difference between any of the groups at any interpulse interval even though there was a tendency to increased inhibition in the SL and DHT groups. Error bars represent SEM.

group, however, exhibitcd a PS size potentiation similar to the control, and significant differences were found between the single-lesioned rats and the DL group (Fig. 13; DHT: $F=5.6, p$ $<0.02$; SL: $F=4.1, p<0.05$ ). In the grafted rats, as was observed when the EPSP slope was measured, PS LTP was significantly reduced compared to the DL rats $(F=6.2 ; p<$ 0.01 ), and these rats were not different from the single-lesioned rats.

\section{Discussion}

The present study demonstrates that 5-HT depletion, in rats that have had partial septal lesion, severely impairs performance in a hippocampus-related spatial memory task (O'Keefe and Nadel, 1978; Morris et al., 1982; Barnes, 1988). This impairment was ameliorated by raphe transplants restricted to the hippocampus, suggesting that serotonergic actions within the a

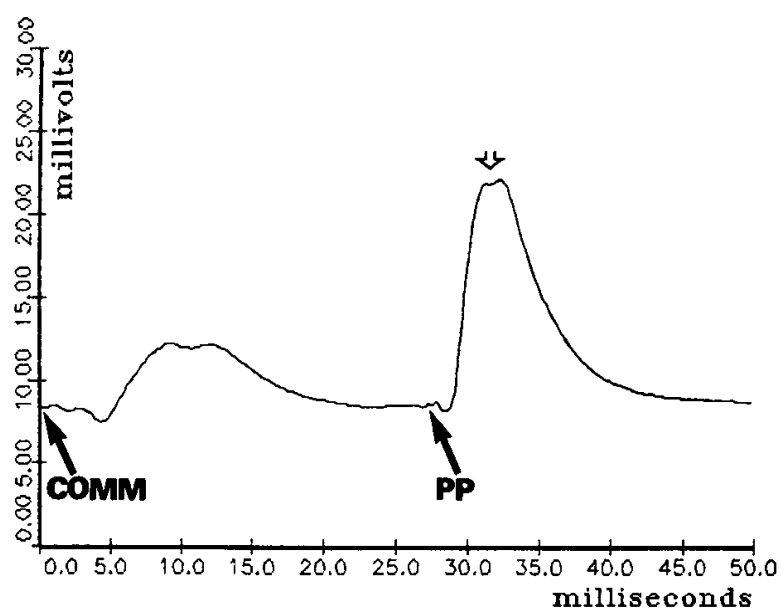

b

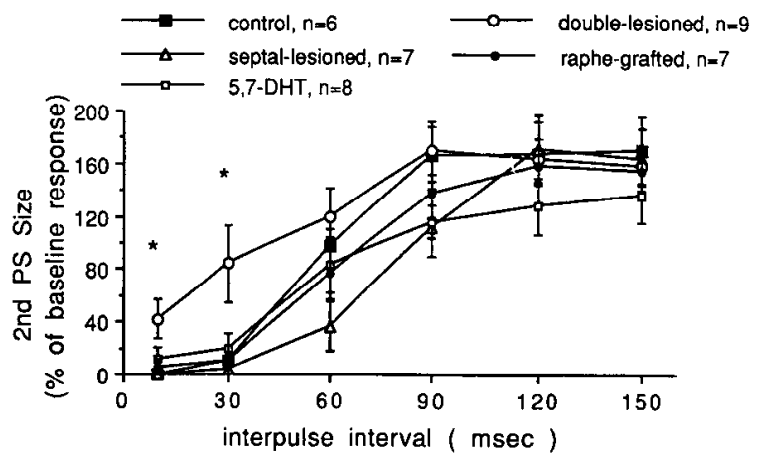

Figure 10. Feedforward inhibition. $a$, The response to PP stimulation $(P P)$, of the same intensity as in Figure $9 a$, is inhibited by priming activation of the commissural pathway (COMM). The open arrow indicates the location of the PS, if not inhibited. $b$, Feedforward inhibition was measured as the decrease in PS size, relative to a baseline response, at different intervals after priming of the commissural connection. Baseline response was at a stimulus intensity that yielded $50 \%$ of the maximal response to PP activation. Asterisks indicate significant differences between control rats and the DL group (ANOVA; 10-msec interpulse interval, $P<0.007 ; 30-\mathrm{msec}$ interpulse interval, $P<0.003$ ). Although not significant, a tendency to an increased inhibition was noticed in the SL group (cf. inhibition at 60-msec interpulse interval). Error bars represent SEM.

hippocampus are important for spatial memory, when the septohippocampal neurotransmission systems are disrupted. 5-HT depletion, under these conditions, also affected a number of physiological processes within the hippocampus. Furthermore, these deficits were also restored by raphe grafts.

\section{Effects on behavior}

5-HT depletion alone had no consistent effect on the performance of rats in spatial behavioral tasks. Treating rats with either $p$-chloroamphetamine (PCA; Altman et al., 1989), p-chlorophenylalanine (PCPA; Richter-Levin and Segal, 1989), or intraraphe injection of 5,7-DHT (Asin et al., 1985) has no effect 


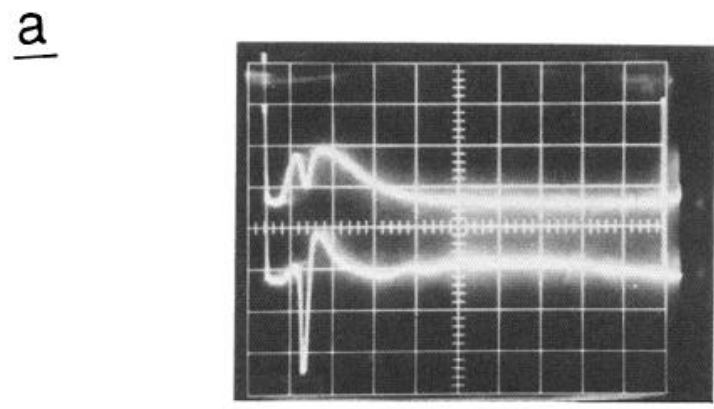

Figure 11. $a$, The response of the GCs to PP stimulation, before (upper trace) and during (lower trace) frequency potentiation. Responses were collected on a digital oscilloscope before and $30 \mathrm{sec}$ after stimulation began. Stimulation consisted of a train of pulses at $7 \mathrm{~Hz}$ for $30 \mathrm{sec}$. Horizontal scale, $5 \mathrm{msec} /$ division; vertical scale, $5 \mathrm{mV} /$ division. $b$, The relative distribution, in each group, between rats that exhibited frequency potentiation and those in which stimulation resulted in a temporal disappearance of the field potential. The DHT group exhibited a significantly reduced ability to exhibit frequency potentiation $\left(\chi^{2}\right.$ test, $\left.p<0.02\right)$. There was no significant difference between any of the other groups and the control group (as tested in separate $\chi^{2}$ tests).

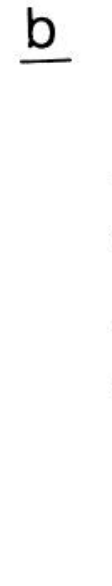

on their performance in either a radial arm maze or a watermaze spatial memory task. Intraventricular injection of 5,7DHT, however, had a mild effect on the performance of rats in a water-maze task similar to that used in the present study (Nilsson et al., 1988).

The contribution of the serotonergic system to the normal functioning of the hippocampus increases under conditions in which other neurotransmission systems are damaged. Severe cholinergic damage is sufficient to disrupt the performance of rats in several learning tasks (Smith, 1988; Segal et al., 1989; Paylor and Rudy, 1990). A partial blockade of the cholinergic system by itself had no noticeable effects on the performance of rats in the present water-maze task (Richter-Levin and Segal, 1989). When combined with such a partial lesion, 5-HT depletion, which had little or no effect of its own, severely reduced the ability of rats to negotiate the maze (Vanderwolf, 1987; Nilsson et al., 1988; Richter-Levin and Segal, 1989). Cortical and hippocampal electrical activities are modulated by both 5-HT and ACh in correlation with behavior (Vanderwolf, 1988). Cholinergic terminals in the rat hippocampus possess serotonergic receptors (Maura and Raiteri, 1986). Furthermore, 5-HT can block facilitatory cholinergic action in the rat hippocampus in vivo (Hong and Krnjevic, 1989). It is thus likely that 5-HT involvement in learning is related to cholinergic transmission in the cortex and hippocampus.

The present study cannot exclude the possible contribution of deficiencies in other septum-originated neurotransmitters to the behavioral deficits observed in the DL rats. The septohippocampal GABAergic connection (Freund and Antal, 1988) is probably as much affected as the cholinergic one and is likely to contribute to the observed behavioral deficits.

We observed a significant improvement in performance of the grafted compared to the DL rats. In a similar study, Nilsson et al. (1990) obtained behavioral recovery only following double transplantation of septal and raphe tissue into double-lesioned rats. There was, however, a difference in the severity of the septal lesions of the two experiments. While we obtained a $50 \%$ reduction in AChE staining, they report a more than $70 \%$ reduction in choline acetyltransferase staining (Nilsson et al., 1988). The severity of the septal lesions was also reflected in the performance of rats in the water maze. In severely lesioned rats, acquisition rate was lower than in controls, and even though these rats eventually achieved escape latencies similar to controls, they were still significantly worse when tested in a spatial probe trial that is equivalent to the quadrant analysis test (Nilsson et al., 1988). In contrast, we found that SL rats did not differ from controls in the reference water-maze task, did not alter search strategy, and performed similarly to controls in the quadrant analysis test. This difference between the two studies signifies the contribution of the serotonergic innervation of the hippocampus in the case of partial septal lesion and suggests that it potentiates the functionality of the remaining septal fibers. However, when these are absent, serotonergic fibers are no longer effective.

The possibility of nonserotonergic effects of the grafts cannot be excluded. To verify the serotonergic nature of the behavioral and physiological effects of such grafts, we would need to modulate pharmacologically serotonergic transmission in these graft- 
a

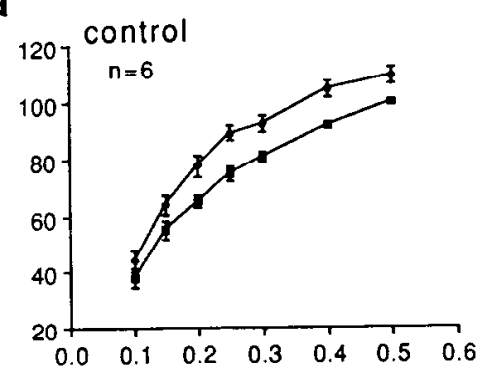

C

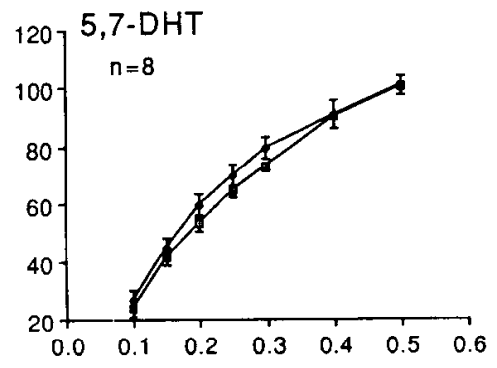

e

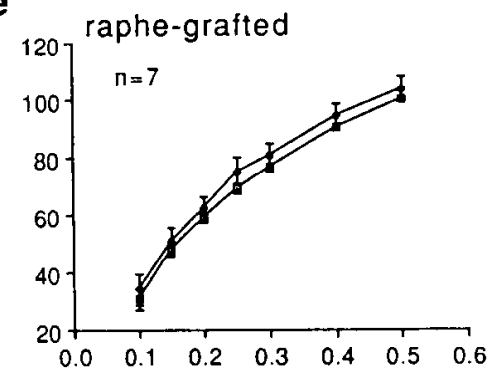

b

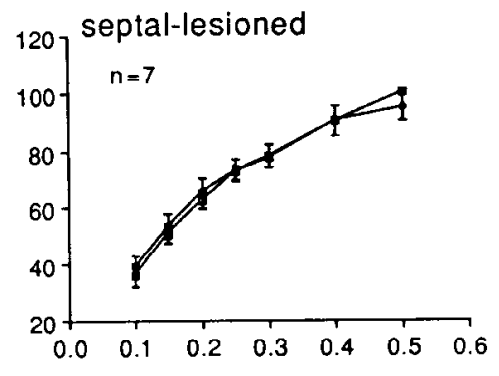

d

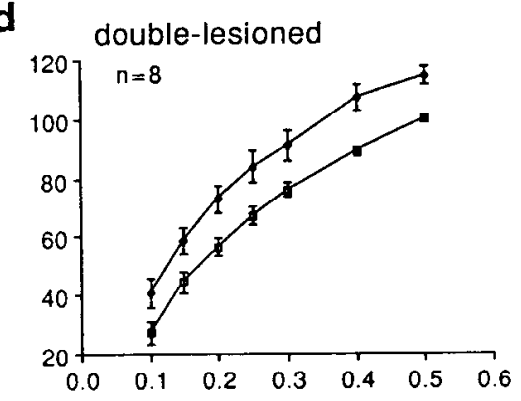

\begin{tabular}{|l|c|c|}
\hline$f$ & $F$ & $p r>F$ \\
\cline { 2 - 3 } & & \\
groups effect: & 5.48 & 0.001 \\
control v.s. DL & 0.97 & 0.33 \\
\hline control v.s. SL & 7.7 & 0.009 \\
\hline control v.s. DHT & 3.2 & 0.08 \\
control v.s. RG & 4.3 & 0.04 \\
\hline DL v.s. DHT & 8.9 & 0.005 \\
\hline DL v.s. RG & 10.6 & 0.003 \\
\hline
\end{tabular}

Figure 12. EPSP slope before and 30 min after tetanic stimulation. $a$, control; $b$. SL; $c$, DHT; $d$. DL; $e$, RG. Vertical axes, percentage of baseline response (at stimulus intensity of $0.5 \mathrm{~mA}$ ); horizontal axes, stimulus intensity (mA). Squares, before; diamonds, 30 min after tetanic stimulation. Error bars represent SEM. $f$, Results from ANO$V A$ for repeated measurements of the increase in EPSP slope. The difference between EPSP slope before and $30 \mathrm{~min}$ after tetanic stimulation was compared over stimulus intensities from 0.1 to 0.5 $\mathrm{mA}$. ed rats. We avoided any pharmacological manipulation in the present study to prevent any possible side effects and long-term drug effects on the electrophysiology and histochemistry of these grafts. An alternative approach would be to compare the effects of raphe grafts to those of a graft from a different origin. We did conduct these experiments elsewhere (G. Richter-Levin and M. Segal, unpublished observations) and found that raphe, but not septal, grafts transplanted into the hippocampus ameliorated the performance deficits of 5-HT-depleted rats, injected with atropine, in a reference-type water-maze task.

The effects of 5-HT depletion on avoidance behaviors seem to reflect differences in the drugs used to elicit depletion of 5-HT (Ogren et al., 1981) and in task details (for review, see Altman and Normile, 1988). In our study, forebrain 5-HT depletion improved the performance of rats in the active avoidance task. Such an improvement may be the result of a general disinhibitory effect on behavior or a result of higher sensitivity to the CS (the tone) and/or to the unconditioned stimulus (US; electric shock). Restoration of serotonergic innervation by raphe grafts in the hippocampus had no effect on active avoidance behavior, suggesting that serotonergic innervation elsewhere in the brain is associated with such behaviors and supporting our assumption that the serotonergic innervation of the hippocampus has a unique role in hippocampus-related behaviors.

\section{Physiology}

LTP is considered a model mechanism for learning and memory (Laroche, 1985). A correlation between the ability to induce LTP and learning abilities has been proposed; in vivo induction of LTP correlates with the ability of rats to acquire a shuttlebox avoidance task (Matthies et al., 1986). A blockade of the NMDA receptor by the specific blocker D,L-2-amino-5-phosphonopentanoic acid blocks both LTP and the ability of rats to perform well in a spatial water-maze task (Morris et al., 1986; Morris, 1989; but see Leung and Desborough, 1988).

Our results, however, failed to support this notion. The performance of the single-lesioned rats in the water maze was better than that of the DL rats, whereas the ability to express LTP was significantly reduced in the single-lesioned rats but was apparently normal in the DL rats. Furthermore, raphe grafts into the hippocampus improved the performance of DL rats in the water maze but suppressed their hippocampal ability to express LTP.

A possible explanation for this discrepancy is that the hippocampi of the DL rats were generally more excitable and that this resulted in a saturation of behaviorally related synaptic plasticity, as was suggested recently (McNaughton et al., 1986). LTP in the hippocampus of rats, evoked prior to acquisition, severely reduced their ability to acquire a spatial task (Mc- 
a

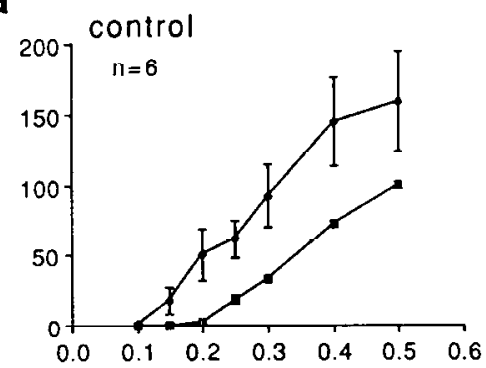

C

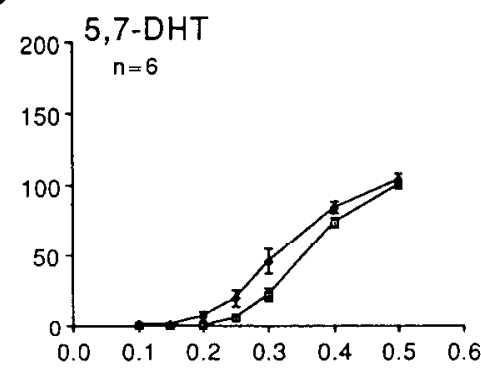

Figure 13. PS size before and $30 \mathrm{~min}$ aftcr tctanic stimulation. $a$, control; $b$, $\mathrm{SL} ; c, \mathrm{DHT} ; d, \mathrm{DL} ; e, \mathrm{RG}$. Vertical axes, percentage of baseline response (at stimulus intensity of $0.5 \mathrm{~mA}$; horizontal axes, stimulus intensity (mA). Squares, before; diamonds, $30 \mathrm{~min}$ after tetanic stimulation. Error bars represent SEM. $f$, Results from ANOVA for repeated measurements of the increase in PS size. The difference in PS size before and $30 \mathrm{~min}$ after tetanic stimulation was compared over stimulus intensities from 0.1 to $0.5 \mathrm{~mA}$.

e b

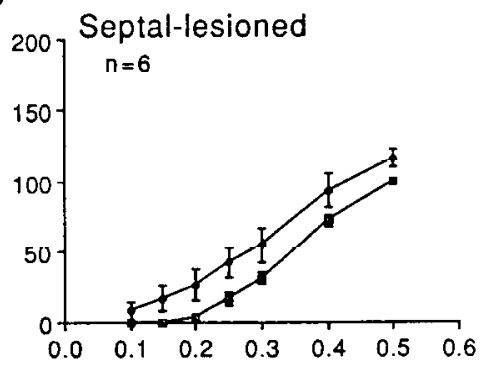

d
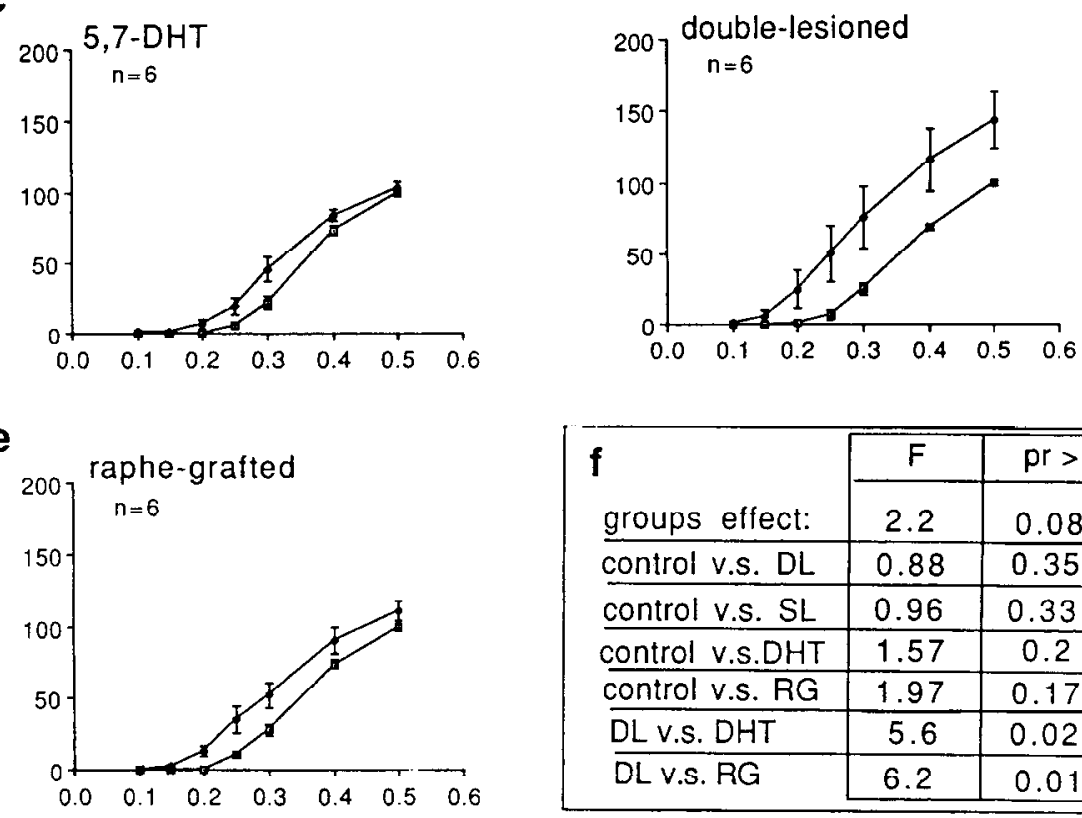

\begin{tabular}{|l|c|c|}
\hline $\mathbf{f}$ & $\mathbf{F}$ & $\mathrm{pr}>\mathrm{F}$ \\
\cline { 2 - 3 } groups effect: & 2.2 & 0.08 \\
\hline control v.s. DL & 0.88 & 0.35 \\
\hline control v.s. SL & 0.96 & 0.33 \\
\hline control v.s.DHT & 1.57 & 0.2 \\
\hline control v.s. RG & 1.97 & 0.17 \\
\hline DL v.s. DHT & 5.6 & 0.02 \\
\hline DL v.s. RG & 6.2 & 0.01 \\
\cline { 2 - 3 }
\end{tabular}

Naughton ct al., 1986), presumably duc to the saturation of the hippocampal synapses and the resulting inability to form specific, behaviorally modulated changes in synaptic efficacy.

The present results support the presence of an enhanced excitability as a result of the combined lesions. Whenever each of the lesions tended to increase inhibition or reduce excitation, this tendency disappeared in the DL rats. Such was the effect on frequency potentiation. A significant reduction was observed in the DHT group that was not observed in the DL group. Such were the effects on LTP, and the most profound was the pronounced reduction in feedforward inhibition that was also significantly different from controls.

The DG contains both GCs and inhibitory interneurons that can modulate the response of the DG to PP stimulation (Lacaille et al., 1989; Sloviter, 1989). Cholinergic and GABAergic cells of the septum innervate the DG. Anatomical evidence suggests that the cholinergic fibers primarily innervate GCs, whereas the GABAergic cells innervate mainly the interneurons (Wheal and Miller, 1980; Freund and Anatal, 1988). Stimulation of the septum has a disinhibitory effect on the DG (Bilkey and Goddard, 1987; Krnjevic et al., 1988). Lesioning the septum is thus expected to reduce excitability of GCs, both by reducing the excitatory cholinergic innervation and by reducing inhibitory GABAergic innervation of the inhibitory interneurons. A tendency toward increased inhibition was noticed in the SL group but was not statistically significant (Fig. 10b). In the abscncc of septum-derived GABAergic inhibition on DG inhibitory interneurons, the serotonergic innervation of the DG may provide this inhibitory modulation of inhibitory interneurons, as is suggested by the excitatory effect of 5-HT-releasing drugs on DG response to PP stimulation (Richter-Levin and Segal, 1988, 1990a). Such a modulation may explain the relatively small effect of septal lesions on hippocampal inhibition. If this assumption is correct, the combined lesions would be expected to result in significantly increased inhibition, due to the release of GABAergic and serotonergic inhibition from the DG inhibitory interneurons, and yet, the opposite was observed. In a similar way, epilepticlike stimulation of the DG results in a selective death of presumably excitatory interneurons and a selective preservation of the inhibitory GABAergic interneurons in the DG. A month after the treatment, a significant reduction in feedback inhibition is detected in such rats (Sloviter, 1989).

A possible explanation of this discrepancy may be that, without inhibition of the inhibitory interneurons, these cells may become hyperactive. This hyperactivity may result in a desensitization or downregulation of the GCs' GABAergic receptors that will eventually result in a decrease in inhibition. Such an interpretation, however, implies that different, short-term alterations will underlie the apparently similar behavioral deficiencies observed in acute (pharmacological) experiments (Van- 
derwolf, 1987; Richter-Levin and Segal, 1989). Such a possibility is currently being investigated. Another possibility is that when the serotonergic and cholinergic-GABAergic innervation is weaker or absent, the relative importance of the remaining neuromodulatory systems, such as that of the noradrenergic system, increases. Noradrenaline (NA) increases DG responsiveness to PP stimulation (for review, see Harley, 1987), and when applied with a subthreshold stimulation, NA may bring about the induction of LTP (Hopkins and Johnston, 1984). These effects can explain the relative excitation observed in the DL rats. Furthermore, this may provide an explanation for both the acute and chronic experiments.

It was recently reported that fornix transection, which abolishes septohippocampal innervation and is also expected to reduce the serotonergic innervation of the dorsal hippocampus, abolishes the ability of the hippocampus to express LTP (Buzsaki and Gage, 1989). The difference between these and our findings is possibly due to the effects of fornix transection on other neurotransmission systems, such as the noradrenergic innervation that reaches the hippocampus via the fornix-fimbria (Harley, 1987). Depletion of NA prevents the induction of LTP (Bliss et al., 1983; Stanton and Sarvey, 1985), and thus, removal of the three main modulatory systems (i.e., NA, 5-HT, and ACh) will reduce the ability of the hippocampus to express LTP.

Depleted forebrain 5-HT in the intact rat leads to reduced LTP (Bliss et al., 1983), though this cannot be repeated in the slice preparation (Stanton and Sarvey, 1985), suggesting that 5 -HT is involved in LTP by affecting extrahippocampal areas, most likely the entorhinal cortex, which receives a dense serotonergic innervation (Azmitia and Segal, 1978; Moore, 1981).

Deficiencies in cognitive abilities, associated with aging and degenerative disorders such as Alzheimer's and Parkinson's discases, were traditionally associated with degeneration of the forebrain cholinergic projection system (Bartus et al., 1982; Coyle et al., 1985). In recent years, accumulating data suggest that in such cases there is a combined reduction in the efficacy of several neurotransmitters and that this may better describe the mechanisms underlying the related mnemonic deficiencies (Baker and Reynolds, 1989; Hertz, 1989; Schlicker et al., 1989). The present study further supports such a notion.

In summary, the serotonergic innervation of the hippocampus is suggested to have a unique role in hippocampus-related behaviors. Reduction in serotonergic modulation of hippocampal electrical activity seems to contribute to the behavioral deficits observed in rats. Restoration of modulation, by raphe grafts into the hippocampus, is suggested to underlie the behavioral recovery observed in these rats.

\section{References}

Altman HJ, Normile HJ (1988) What is the nature of the role of the serotonergic nervous system in learning and memory: prospects for development of an effective treatment strategy for senile dementia. Neurobiol Aging 9:627-638.

Altman HJ, Ogren SO, Berman RF, Normile HJ (1989) The effects of $p$-chloroamphetamine, a depletor of brain serotonin, on the performance of rats in two types of positively reinforced complex spatial discrimination tasks. Behav Neural Biol 52:131-144.

Andersen P, Lomo T (1967) Control of hippocampal output by afferent volley frequency. Prog Brain Res 27:400-412.

Asin KE, Wirtshafter D, Fibiger HC (1985) Electrolytic, but not 5,7dihydroxytryptamine, lesions of the nucleus medianus raphe impair acquisition of radial maze task. Behav Neural Biol 44:415-424.

Austin KB, Bronzino JD, Morgane PJ (1989) Paired-pulse facilitation and inhibition in the dentate gyrus is dependent on behavioral state. Exp Brain Res 77:594-604.

Azmitia EC, Segal M (1978) An autoradiographic analysis of the differential ascending projections of the dorsal and median raphe nuclei in the rat. J Comp Neurol 179:641-668.

Baker GB, Reynolds GP (1989) Biogenic amines and their metabolites in Alzheimer's disease: noradrenaline, 5-hydroxytryptamine and 5-hydroxyindole-3-acetic acid depleted in hippocampus but not in substantia innominata. Neurosci Lett 100:335-339.

Barnes CA (1988) Spatial learning and memory processes: the search for their neurological mechanisms in the rat. Trans Neurosci 11:163169.

Bartus RT, Dean RL, Beer B, Lippa AS (1982) The cholinergic hypothesis of geriatric memory dysfunction. Science 217:408-417.

Biegon $\Lambda$, Wolff M (1986) Quantitative histochemistry of acctylcholinesterase in rat and human brain postmortem. J Neurosci Methods $16: 39-45$.

Bilkey DK, Goddard GV (1987) Septohippocampal and commissural pathways antagonistically control inhibitory interneurons in the dentate gyrus. Brain Res 405:320-325.

Bliss TVP, Goddard GV, Riives M (1983) Reduction of long-term potentiation in the dentate gyrus of the rat following selective depletion of monoamines. J Physiol (Lond) 334:475-491.

Bramham CR, Srebro B (1989) Synaptic plasticity in the hippocampus is modulated by behavioral state. Brain Res 493:74-86.

Buzsaki G, Gage FH (1989) Absence of long-term potentiation in the subcortically deafferented dentate gyrus. Brain Res 484:94-101.

Coyle JT, Price DL, DeLong MR (1985) Alzheimer's disease: a disorder of cortical cholinergic innervation. In: Ncuroscience (Abelson PH, Butz E, Snyder S, eds), pp 418-431. Washington, DC: American Association for the Advancement of Science.

Freund TF, Antal M (1988) GABA-containing neurons in the septum control inhibitory interneurons in the hippocampus. Nature 336:170 173.

Gross-Isseroff R, Biegon A (1988) Autoradiographic analysis of ${ }^{3} \mathrm{H}-$ imipramine binding in the human brain postmortem: effects of aging and alcohol. J Neurochem 51:528-534.

Harley CW (1987) A role for norepinephrine in arousal, emotion and learning?: limbic modulation by norepinephrine and the Kety hypothesis. Prog Neuropsychopharmacol Biol Psychiatry 11:419-458.

Hertz L (1989) Is Alzheimer's disease an anterograde degeneration, originating in the brainstem, and disrupting metabolic and functional interactions between neurons and glial cells? Brain Res Rev 14:335353.

Hong Y, Krnjevic K (1989) Serotonin block the facilitatory action of muscarinic and nicotinic agents in the hippocampus in vivo. Can J Physiol Pharmacol 69:47-53.

Hopkins WF, Johnston D (1984) Frequency-dependent noradrenergic modulation of long-term potentiation in the hippocampus. Science 226:350-351.

Isseroff A, Lancet D (1985) An inexpensive microcomputer-based image-analysis system: novel application to quantitative autoradiography. J Neurosci Methods 12:265-275.

King FA (1958) Effects of septal and amygdala lesions on emotional behavior and conditioned avoidance responses in the rat. J Nerv Ment Dis 126:57-63.

Krnjevic K, Ropert N, Casullo J (1988) Septohippocampal disinhibition. Brain Res 438:182-192.

Lacaille JC, Kunkel DD, Schwartzkroin PA (1989) Electrophysiological and morphological characterization of hippocampal interneurons. In: The hippocampus: new vistas (Chan-Palay V, Kohler C, eds), pp 287-305. New York: Liss.

Laroche $S$ (1985) What can the long-term potentiation procedure tell us about the neural mechanisms of learning and memory? In: Advances in behavioral biology, Vol 28 , Brain plasticity, learning and memory (Will BE, Schmitt P, Dalrymple-Alford JC, eds), pp 139155. New York: Plenum.

Leung LS, Desborough KA (1988) APV, an $N$-methyl-D-aspartate receptor antagonist, blocks the hippocampal theta rhythm in behaving rats. Brain Res 463:148-152.

Matthies H, Ruethrich H, Ott T, Matthics HK, Mathies R (1986) Low frequency perforant path stimulation as a conditioned stimulus demonstrates correlations between long-term synaptic potentiation and learning. Physiol Behav 36:811-821.

Maura G, Raiteri M (1986) Cholinergic terminals in rat hippocampus 
possess $5-\mathrm{HT}_{1 \mathrm{~B}}$ receptors mediating inhibition of acetylcholine release. Eur J Pharmacol 129:333-337.

McNaughton BL, Barnes CA, Rao G, Baldwin J, Rasmussen M (1986) Long-term enhancement of hippocampal synaptic transmission and the acquisition of spatial information. J Neurosci 6:563-571.

Moore RY (1981) The anatomy of central serotonin neuron system in the rat brain. In: Serotonin-neurotransmission and behavior (Jacobs BL, Gelperin A, eds), pp 35-71. Cambridge, MA: MIT Press.

Morris RGM (1989) Synaptic plasticity and learning: selective impairment of learning in rats and blockade of long-term potentiation in vivo by the $N$-methyl-D-aspartate receptor antagonist AP5. J Neurosci 9:3040-3057.

Morris RGM, Garrud P, Rawlins JNP, O'Keefe J (1982) Place navigation impaired in rats with hippocampal lesions. Nature 297:681683.

Morris RGM, Anderson E, Lynch GS, Baudry M (1986) Selective impairment of learning and blockade of long-term potentiation by an $N$-methyl-D-aspartate receptor antagonist, AP5. Nature 319:774-776.

Nilsson OG, Strecker RE, Daszuta A, Bjorklund A (1988) Combined cholinergic and serotonergic denervation of the forebrain produces severe deficits in a spatial learning task in the rat. Brain Res 453:235246.

Nilsson OG, Brundin P, Bjorklund A (1990) Amelioration of spatial memory impairment by intrahippocampal grafts of mixed septal and raphe tissue in rats with combined cholinergic and serotonergic denervation of the forebrain. Brain Res 515:193-206.

Ogren SO, Fuxe K, Archer T, Hall H, Holm AC, Kohler C (1981) Studies on the role of central 5-HT neurons in avoidance learning: a behavioral and biochemical analysis. In: Serotonin, current aspects of neurochemistry and function (Haber B, Gabay S, Issidorides MR, Alivisatos SGA, eds), pp 681-705. New York: Plenum.

O'Keefe J, Nadel L (1978) The hippocampus as a cognitive map. Oxford: Clarendon.

Paylor R, Rudy JW (1990) Cholinergic receptor blockade can impair the rat's performance on both the place learning and cued versions of the Morris water task: the role of age and pool wall brightness. Behav Brain Res 36:79-90.

Richter-Levin G, Segal M (1988) Serotonin releasers modulate reactivity of the rat hippocampus to afferent stimulation. Neurosci Lett 94:173-176.

Richter-Levin G, Segal M (1989) Spatial performance is severely im- paired in rats with combined reduction of serotonergic and cholinergic transmission. Brain Res 477:404-407.

Richter-Levin G, Segal M (1990a) Effects of serotonin releasers on dentate granule cell excitability in the rat. Exp Brain Res 82:199-207.

Richter-Levin G, Segal M (1990b) Grafting of midbrain neurons into the hippocampus restores serotonergic modulation of hippocampal activity in the rat. Brain Res 521:1-6.

Schlicker E, Betz R, Gothert M (1989) Investigation into the agedependence of release of serotonin and noradrenaline in the rat brain cortex and the autoreceptor-mediated modulation of release. Neuropharmacology 28:811-815.

Segal M, Azmitia E (1986) Fetal raphe neurons grafted into the hippocampus develop normal adult physiological properties. Brain Res 364:162-166.

Segal M, Greenberger V, Pearl E (1989) Septal transplants ameliorate spatial deficits and restore cholinergic functions in rats with a damaged septo-hippocampal connection. Brain Res 500:139-148.

Sharp PE, McNaughton BL, Barnes CA (1989) Exploration-dependent modulation of evoked responses in fascia dentata: fundamental observations and time course. Psychobiology 17:257-269.

Sloviter RS (1989) Chemically defined hippocampal interneurons and their possible relationship to seizure mechanisms. In: The hippocampus: new vistas (Chan-Palay V, Kohler C, eds), pp 443-461. New York: Liss.

Smith G (1988) Animal models of Alzheimer's disease: experimental cholinergic denervation. Brain Res Rev 13:103-118.

Squire LR, Zola-Morgan S (1988) Memory: brain systems and behavior. Trans Neurosci 11:170-175.

Stanton PK, Sarvey JM (1985) Depletion of norepinephrine, but not serotonin, reduces long-term potentiation in the dentate gyrus of rat hippocampal slices. J Neurosci 5:2169-2176.

Vanderwolf $\mathrm{CH}$ (1987) Near-total loss of 'learning' and 'memory' as a result of combined cholinergic and serotonergic blockade in the rat. Behav Brain Res 23:43-57.

Vanderwolf CH (1988) Cerebral activity and behavior: control by central cholinergic and serotonergic systems. Int Rev Neurobiol 30: 225-340.

Wheal HV, Miller JJ (1980) Pharmacological identification of acetylcholine and glutamate excitatory systems in the dentate gyrus of the rat. Brain Res 182:145-155. 